\title{
BUEN VIVIR, SISTEMA ESCOLAR Y EDUCACIÓN FÍSICA EN CHILE: UNA REALIDAD POR CONSTRUIR
}

Good living, school system and physical education in chile: a reality to build A boa vida, do sistema escolar e educação física no chile: uma realidade para construir

\author{
Antonio Andrés López Pérez \\ Pontificia Universidad Católica de Valparaíso. Fono: +56988329207. Correo \\ electrónico: Antonio.lopez@pucv.cl
}

\begin{abstract}
Resumen
Al mirar la realidad educativa de nuestro país, nos podríamos preguntar ¿Cómo nos hacemos cargo como profesores, de las diversas falencias que presenta nuestro sistema, si nuestro propio actuar ha dejado de tener cierta autonomía en el quehacer educativo? A esto podemos sumar, que los actuales cambios políticos y económicos, también han generado un descontento y un malestar docente que se proyecta desde la post-dictadura. Desde la mirada de la Educación física, se plantean otras interrogantes: ¿Por qué el currículum escolar está tan sobrecargado de elementos, si tan solo aprendemos algunos de ellos? ¿Son nuestras prácticas espacio de reflexión y de un hacer en torno a una lógica distinta a la tradicional? ¿Crecemos económicamente por crecer o para satisfacer las necesidades humanas? El problema no es lo que hago, sino la intencionalidad, ya que la Escuela no ha existido siempre, pero la Educación sí. Pareciera ser que esta última, en el caso de las culturas antiguas era de mejor calidad que la que tenemos hoy en día, con una visión de comunidad que busca un objetivo: La sustentabilidad. Esta no se logrará hasta que seamos conscientes de nuestra propia especie y reconociendo que somos una parte del mundo que nos rodea. Si bien la profesión docente no ha sido valorada como corresponde, la cambiante realidad de nuestros días nos obliga a reflexionar acerca del tipo de docente deseable en nuestras salas de clases, logrando que los estudiantes participen activamente de la sociedad que los rodea. Palabras Claves: buen vivir; sistema escolar; educación física.
\end{abstract}

\footnotetext{
Abstract

Buen vivir, sistema escolar y educación física en chile: una realidad por construir
} 
Looking at the educational reality of our country, as teachers we may ask: how do we make ourselves accountable for the various deficiencies our educational system has, when we have become less autonomous regarding educational work? We may also add, that the current political and economic changes have caused discomfort and discontent among teachers which originates after the military dictatorship. From the physical education point of view, other questions arise: Why is the school curriculum full of elements if we only learn a part of them? Is our teaching practice the place to reflect and take action regarding a nonconventional logic? Do we economically grow just to grow or to satisfy human needs? The problem is not what I do, but the purpose, since the school has not always existed, but education has. It seems to be that the latter, in the case of ancient civilizations, had better quality than the one we have nowadays, with a community idea that seeks an objective: sustainability. This will not be achieved until we are aware of our own kind and acknowledge that we are a part of the world around us. Even though the teaching profession has not been valued as it should, current changing reality forces us to reflect upon the ideal teacher type for our classrooms; managing students taking an active part of the world they live in.

Key words: good living; school system; physical education

\section{Resumo}

Ao olhar a realidade educativa do nosso pais, poderíamos perguntar-nos: Como fazer-nos cargo como professores, das diversas falências que se apresentam no nosso sistema, se nosso próprio atuar a deixado de ter certa autonomia no que fazer educativo? Nisto podemos somar, que as atuais modificações políticas e econômicas, também ao gerado um desconforto docente que se projeto desde a pos-ditadura. Desde o olhar da educação física, se planteia outros interrogantes: Porque o curriculum escolar esta tão sobrecarregada de elementos, se tão só aprendemos alguns deles? São nossas praticas, espaço de reflexão e de um fazer ao redor de uma lógica diferente a tradicional? Achamos economicamente que por crescer ou para satisfazer as necessidades humanas? O problema não é o que faço, senão na intencionalidade, já que a escola não a existido sempre, mas a educação sim. Pareceria ser que esta ultima, no caso das culturas antigas é de melhor qualidade que a que temos hoje 
em dia, com uma visão de uma comunidade que procura um objetivo: A sustentabilidade. Isto não se consegui ate que sejamos consciente de nossa própria espécie e reconhecendo que somos uma parte do mundo que nos rodeia. Por mais que nossa profissão docente não tem sido valorada como corresponde, a mudança da realidade de nossos dias nos obriga a refletir sobre o tipo de docente desejável em nossa sala de aula, logrando que os estudantes participem ativamente na sociedade que os rodeia.

Palavras chaves: bom viver; sistema escolar; educação fisica. 
La educación física en Chile, ha pasado por innumerables transformaciones en el último tiempo y hoy incluso su nombre lleva un apellido: "Salud". En la escuela, se denomina Educación física y salud y la preocupación por mantener un "completo estado de bienestar físico, psíquico y social” establecida por la Organización Mundial para la Salud (OMS, 1960), ya no sólo entendiéndola como lo ausencia de enfermedad, ha estado presente desde diversas miradas y sigue siendo un tema recurrente a partir de los cambios políticos, económicos y sociales, junto a cambios epidemiológicos (Wolff, 2002) que posicionan a las enfermedades crónicas no transmisibles (ECNT) en el ojo del huracán y en una de las prioridades para los gobiernos de turno.

Es así, que diversos autores no sólo plantean que la salud se relaciona con la ausencia de enfermedad, sino que es un concepto multidimensional. De esta forma, Perea (1992), destaca la salud como el "conjunto de condiciones y calidad de vida que permita a la persona desarrollar y ejercer todas sus facultades en armonía y relación con su propio entorno". Corbella (Corbella, 1993) definió la salud como "una manera de vivir cada vez más autónoma, más solidaria y más gozosa” y Rodríguez (Rodríguez, 2010), la define como "el proceso por el que el hombre desarrolla al máximo sus capacidades, tendiendo a la plenitud de su autorrealización personal y social”. Si analizamos estas concepciones de la salud encontraremos que se muestra notablemente vinculado al concepto de calidad de vida sana (Sánchez Bañuelos, 1996).

Al parecer para muchos salud, calidad de vida y buen vivir son sinónimos, pero a pesar de estar relacionados, nos referimos a cosas distintas. El primero lo identificamos al igual que la OMS como un estado que abarca diversas aristas del ser humano; La calidad de vida, entendida como la combinación de componentes objetivos y subjetivos, es decir, definida como la calidad de las condiciones de vida de una persona junto a la satisfacción que ésta experimenta (Gómez \& Sabeh, 2001); Y el Buen vivir, lo entenderemos como la capacidad de lograr un equilibrio entre las necesidades fundamentales de la humanidad y los recursos disponibles para satisfacerlas (De la Cuadra, 2015).

De esta forma, al tratar de dilucidar el problema, nos podemos plantear tres interrogantes: ¿Qué? ¿Cómo? y ¿Para qué?, siendo la salud, calidad de vida y buen vivir las 
respuestas. Pero, ¿las respuestas se dan en orden secuencial? ¿Existen intereses políticos que permiten mirar el problema y la solución con matices distintos? ¿Es en realidad la escuela, el punto de partida para comenzar a generar cambios? ¿De qué manera nos hacemos cargo del buen vivir y sus implicaciones para la educación chilena?

Como menciona González (González et. al, 2007), "La sociedad actual demanda la necesidad de incorporar a la cultura y a la educación, aquellos conocimientos, destrezas y capacidades, que se relacionan con el cuerpo y la actividad motriz, contribuyendo de forma armónica al desarrollo personal y a una mejora de la calidad de vida”. Este autor también menciona, la importancia de que todas las alumnas y alumnos adquieran hábitos saludables, con la finalidad de que posibiliten sentirse satisfechos con su propia identidad corporal, la cual será vehículo de expresión y comunicación consigo mismo y con los demás. Esto último permitirá el disfrute del ocio y del tiempo libre, lo que les permitirá una mejora en su calidad de vida.

Pero, no sólo la escuela incide en estos procesos de adquisición de hábitos, ya que la familia asume un rol preponderante, ya que es en ese núcleo, donde se satisfacen las necesidades que están en la base de la conservación, fomento y recuperación de la salud. Cumple funciones importantes en el desarrollo biológico, psicológico y social de cada persona, y es la encargada de la formación y desarrollo de la personalidad, asegurando, junto a otros grupos sociales, la socialización y educación, para su inserción en la vida social y la trasmisión de valores culturales, ético - morales y espirituales (Louro, 2003).

Por lo tanto, la escuela y la familia son los ambientes educativos, que generarán la mayor influencia en la adquisición de hábitos y estilos de vida, que se irán consolidando a lo largo de la infancia y la adolescencia. Durante esta última etapa, los jóvenes tienden a presentar cambios actitudinales y psicológicos, que los hace adoptar distintas conductas y hábitos, por lo que se considera una etapa indicada para promover un estilo de vida saludable. Es así que surge otra pregunta ¿Cómo nos hacemos cargo como profesores, si nuestro propio actuar ha dejado de tener cierta autonomía en el quehacer educativo? A esto podemos sumar, que los actuales cambios políticos y económicos, también han generado un descontento y un malestar docente que se proyecta desde la post-dictadura. 
La realidad de Chile y el descontento social no parecen ser tan al azar, ya que en treinta años se han ido privatizando casi la totalidad de los servicios básicos, replicando políticas neoliberales, influyendo incluso en áreas tan importantes como la salud y la educación. De esta manera, se ha generado una dualidad entre quienes tienen los recursos para pagar por mejores servicios y aquellos que sólo pueden acceder a servicios de menor calidad, alejándonos de la práctica de un buen vivir. En el caso puntual de la educación, Chile implementó el diseño de un sistema de financiamiento basado en subsidios estatales, independiente si los establecimientos tenían administración pública o privada, se privatizaron un alto porcentaje de escuelas, se generó una competencia de mercado entre ellas, se ha ido desmantelando la educación pública y el lucro en diversos establecimientos, era o es algo cotidiano por parte de los sostenedores de algunos colegios. El Estado había dejado en manos de las familias los pagos y se podía seleccionar a los estudiantes. Todo esto, fue promulgado en una ley, en el período de la dictadura militar y ha generado graves problemas de calidad educacional, inequidad y un inédito fenómeno de segmentación sociocultural entre las escuelas chilenas, que hasta el día de hoy vemos a diario y que acentúa el malestar docente, por las diversas e injustas condiciones de trabajo, entre el sistema público y privado.

Esto último, genera una sensación tensa de bienestar-malestar, entre los profesionales de la educación, que tienen que lidiar con: nuevas demandas laborales, básicamente por el cambio en los enfoques pedagógicos deseados; presión por los rendimientos estandarizados; aumento en las brechas de desigualdad socioeducativa; incorporación de nuevos sectores populares a la escolarización; introducción de la lógica gerencial en la escuela; sobrecarga de trabajo; falta de apoyo desde instituciones externas a la escuela (Cornejo, 2009). Pero lo más importante, la desvalorización social de nuestra profesión, siendo el proyecto de Ley de Carrera Profesional Docente, un claro ejemplo de cómo se ha ido devaluando nuestra labor. Para las y los docentes se trata de una necesidad impostergable de mejorar las condiciones laborales, salariales y contractuales en que se desarrolla la profesión docente, transformando con ello la valoración social de ésta y, por qué no, poniendo en tela de juicio la lógica mercantil en que opera el mundo de la política educativa (Redón, 2015). 
La escuela nos condiciona y/o dirige el trabajo que nosotros queremos llevar a cabo y se ha apoderado del proceso educativo, pero cuidado, la educación no se limita a la escuela, se utiliza para seguir reproduciendo ciertas lógicas. La escuela no ha existido siempre, pero la Educación sí. Pareciera ser que esta última, en el caso de las culturas antiguas era de mejor calidad que la que tenemos hoy en día. En las políticas públicas se habla mucho de los establecimientos educacionales, pero se habla poco de Educación en sí, no se dialoga sobre principios educativos, si no sobre principios escolares, esto determina lo que como profesores de educación física, vamos a hacer en la promoción de hábitos de vida saludable. Tenemos que aprender a convertir el saber disciplinar en conocimiento científico, ese será el desafío y aprender a convivir con la falta de tiempo.

La Educación Física es un factor clave en el proceso de socialización de las personas, contribuye a su calidad de vida y desempeña un papel muy importante en el desarrollo global de la persona. Cada una de nuestras acciones cotidianas, van acompañadas de la acción física, pero la evolución de ésta, dependerá de aspectos sociales, culturales y económicos, propios de una sociedad, en un espacio y tiempo determinado. Desde nuestro papel de adultos, hemos de facilitarles a los estudiantes, experiencias que les ayuden a vivir y entender la pluralidad de la actividad física, la importancia de un buen estado de salud, que conlleva a una mejor calidad de vida y que se sustenta en la práctica del buen vivir.

El bienestar humano, tiene que ver con el cómo yo me siento biológicamente, psicológicamente y socialmente. El estado de bienestar no tiene que ver con el cómo se ve mi cuerpo, si no con cómo me siento yo en relación a todas las dimensiones. Debemos dejar de mirar este estado desde una sola mirada. Ya que las políticas públicas evidencian simplicidad en la manera de abordar el problema. Se está estigmatizando a la población y tenemos que entender que la ciencia ha cometido algunos errores como por ejemplo: Generar escalas para situarnos en un determinado lugar. Se ha generado una visión lineal y dicotómica que es contrario al equilibrio dinámico. Un claro ejemplo son las causas de la obesidad en la mayoría de los países cuyas políticas apuntan a un deterioro de la dieta, a la falta de actividad física y a la ignorancia en educación nutricional. Desde el Cartesianismo del siglo XVII, hemos entendido la realidad de la dicotomía, "pienso y luego existo" y esta 
visión nos ha llevado a construir políticas públicas limitadas y limitantes que nos indican que debemos mejorar la dieta, hacer más actividad física y fomentar la educación nutricional. Pero este paradigma simplificador de la realidad y basado en el sistema político, social y económico del país, ha dejado tranquilo a los grandes empresarios, pero insatisfechos a la mayoría de la población.

¿Por qué el currículum escolar está tan sobrecargado de elementos, si tan solo aprendemos no más de diez cosas que son necesarias? ¿Cuál podría ser una lógica diferente para trabajar en Educación Física (EFI) en nuestro país? ¿Son nuestras prácticas espacio de reflexión y de un hacer en torno a una lógica distinta a los planes y programas? ¿Crecemos económicamente por crecer o para satisfacer las necesidades humanas? El problema no es lo que hago, sino la intencionalidad de lo que hago. Todo esto tiene que ver con el bienestar y nos permite preguntarnos si existen realidades en que se aplique el buen vivir en la educación.

¿Cómo pensar en una EFI distinta? Un ejemplo sería ver la Educación como un proceso de creación de relaciones posibles. En cambio en el colegio, se establece una relación ya existente. Se aprende más en espacios no formales (Calvo, 2010). Entonces debemos salir de la burbuja en la que nos encontramos y ver el proceso de enseñanzaaprendizaje desde otro prisma. Es así como Bolivia o Ecuador, tienen una gran cantidad de experiencias, en torno al buen vivir en el caso de Sudamérica y otros países también han incursionado en nuevas propuestas.

Es el caso de las Escuelas como comunidades de aprendizaje, en España, implican a todas las personas que de forma directa o indirecta influyen en el aprendizaje y el desarrollo de las y los estudiantes, incluyendo a profesores, familiares, amigos y amigas, vecinos y vecinas del barrio, miembros de asociaciones y organizaciones vecinales y locales, personas voluntarias, entre otras. La transformación de los centros educativos en Comunidades de Aprendizaje se basa en aquellos procedimientos que contribuyen a la superación del fracaso escolar y los problemas de convivencia en todo el mundo y se siguen, entre otras, las siguientes fases: 1.- Sensibilización; 2.- Toma de decisión; 3.- El sueño; 4.- Selección de prioridades; 5.- Planificación (Díez, 2010). Todo esto con la 
finalidad de aprovechar todos los recursos existentes en la Comunidad escolar y así unir los esfuerzos, para alcanzar los objetivos comunes.

Algunos países de América del Sur, sustentan desde una visión indígena y de los pueblos originarios el buen vivir, reconociéndolo como una modificación de la estructura del Estado. Constituye un modelo económico alternativo al capitalismo y busca la armonía entre las personas y la naturaleza. Para estas comunidades el buen vivir es comunitario y responsabilidad de cada miembro y el bien común es tarea del Estado. Pero esta visión de comunidad, busca un objetivo: La sustentabilidad. Esta no se logrará hasta que seamos conscientes de nuestra propia especie y reconociendo que somos una parte del mundo que nos rodea. Si no somos capaces de entender que estamos relacionados e interconectados con otros seres vivos y que tenemos una conexión con nuestro planeta, el buen vivir quedará como una linda utopía que no podremos llevar a la práctica.

De esta manera, nuestra sociedad al hablar de buen vivir, cuestiona y confronta el concepto de desarrollo que hasta ahora hemos asumido, también cuestiona la existencia de un Estado en retirada y a la sociedad cuya referencia de vida es el consumo, la sobrevivencia individual, la acumulación, el tener éxito individual en desmedro del bien colectivo.

¿Qué debemos hacer para construir una Educación Física basada en el buen vivir? Al parecer tenemos que reflexionar y descubrir cuáles son las necesidades que nos demanda la sociedad y qué recursos tenemos para afrontar este escenario que nos posiciona como una disciplina que podrá dar respuesta a los múltiples problemas de salud que aquejan a la sociedad. ¿Seremos capaces como profesionales de buscar el equilibrio y de actuar pese a todas las dificultades que se nos interpongan? ¿Tendremos las ganas de soñar y de querer ser mejores profesionales, para cambiar el mundo? ¿Cómo se define a un buen profesor? La mayoría de los países, incluido Chile, tiene definidas las competencias que debe tener un buen docente, las que aplican en los programas de evaluación o de acreditación de excelencia docente. No basta con manejar bien la disciplina, sino con guiar la búsqueda del conocimiento de manera de garantizar que todos los alumnos, independiente de su realidad, 
aprendan. En este sentido, un buen profesor debe ir de lo general a lo particular, mostrando mayor complejidad de manera paulatina.

Si bien la profesión docente no ha sido valorada como corresponde, la cambiante realidad de nuestros días nos obliga a reflexionar acerca del tipo de docente deseable en nuestras salas de clases, significa entregar más de lo que actualmente entregan algunos profesores, o sea, hacer que los estudiantes participen activamente de la sociedad que los rodea. No debemos olvidar, que si necesitamos cambiar o arreglar el mundo, debemos partir por la educación, esa es la base de cualquier cambio, pero para eso necesitamos cambiar nuestra manera de pensar, el cómo nos relacionamos con el medio, ser personas íntegras, con una base sólida de conocimientos y valores, pero entendiendo que el proceso educativo, ya no sólo se vive en la escuela, sino que debemos traspasar las barreras del aula e introducirnos en el ambiente de cada estudiante. La creatividad, el trabajo en equipo, son factores importantes a la hora de trabajar (Valls, 2003), ya no más la estructura de jerarquía, ahora se avanza con una estructura en bloque, de grupos, donde todos los grupos son independientes, pero unidos de alguna forma. Trabajar en equipo, expresar emociones, y saber conocer las emociones de quienes nos rodean, nos permitirá seguir trabajando en conjunto, relacionándonos y entendiéndonos, para poder llevar a cabo las ideas que surgen de cada uno de los integrantes del proceso educativo.

El camino es largo, pero el compromiso social nos pide seguir adelante. Como dice (Galeano, 1996), citado por (Blázquez, 2001, p.104): “Ella está en el Horizonte, me acerco dos pasos, ella se aleja dos pasos. Camino diez pasos y el horizonte se corre diez pasos más allá. Por mucho que camine, nunca la alcanzaré. ¿Para qué sirve la utopía? Para eso sirve: Para caminar". ¿Por qué no nos transformamos nosotros, los profesores de Educación Física, que siempre hemos estado relacionados con el movimiento, la actividad y la acción, los que comencemos a caminar?

"Si la escuela ha envejecido tanto, si los profesores como colectivo hemos perdido un tren, es tal vez porque hemos centrado nuestra labor en enseñar y no en aprender... el aprendizaje como estilo de vida es una mentalidad, es una forma de vivir la vida y de ir por el mundo". (Bazarra, 2005). Quizás ese sea el aprendizaje a futuro que debiésemos 
conseguir. Ya vendrá el momento de ponerlo en práctica y de descubrir que en el transcurso de nuestro aprendizaje, no sólo hubo personas que nos enseñaron, sino que también existieron aquellos que aprendieron junto a nosotros.

La educación encierra un tesoro, como dice Delors, pero depende de nosotros como profesionales de la pedagogía, el cómo masificaremos esta riqueza, acompañando en el camino a quienes deseen conocer, hacer y convivir en un estado de Buen vivir.

¿Buen vivir, sistema escolar y educación física en Chile: una realidad por construir?

\section{Referencias bibliográficas}

Bazarra, L. (2005). Ser Profesor y Dirigir Profesores en Tiempos de Cambio. Narcea, S.A. De Ediciones. Madrid. España.

Blázquez, D. (2001). La Educación Física. Inde Publicaciones. Barcelona. España.

Calvo, C., \& Elizalde, A. (2010). Educación: creación de nuevas relaciones posibles. Polis (Santiago), 9(25), 7-15.

Corbella, M. (1993). Educación para la salud en la escuela. Aspectos a evaluar desde la educación física. Apuntes: Educación Física y Deportes, (Pág 55-61).

Cornejo, R. (2009). Condiciones de trabajo y bienestar/malestar docente en profesores de enseñanza media de Santiago de Chile. Educação \& Sociedade, 30(107), 409-426.

De la Cuadra, F. (2015). Buen Vivir: ¿Una auténtica alternativa post-capitalista?. Polis (Santiago), 14(40), 7-19.

Díez, J.; Flecha, R. (2010). Comunidades de Aprendizaje: un proyecto de transformación social y educativa. Monográfico sobre Comunidades de Aprendizaje. Revista Interuniversitaria de Formación del Profesorado continuación de la antigua Revista de Escuelas Normales. 67, 24.

Gómez, M., \& Sabeh, E. (2001). Calidad de vida. Evolución del concepto y su influencia en la investigación y la práctica. Salamanca: Instituto Universitario de Integración en la Comunidad, Facultad de Psicología, Universidad de Salamanca. 
González y cols (2007): (Artículo de revista de ciencias de la actividad física y el deporte, Habilidad Motriz $\mathrm{N}^{\circ}$ 28, COLEF de Andalucía, La instauración de estilos de vida saludables desde el área de educación física: la importancia de hábitos de actividad físico-deportiva, González Jurado José Antonio, Manzano Moreno José Ignacio, Pradas De Lafuente Francisco, 2007, Página 5-10).

Louro Bernal, I. (2003). La familia en la determinación de la salud. Revista Cubana de Salud Pública, 29(1), 48-51.

Perea, R. (1992). Educación para la salud en las materias transversales como criterio de calidad educativa. III Jornadas sobre la LOGSE. Granada: Proyecto Sur de Ediciones. (Pág 114).

Redón, S., Angulo, F., \& Serra L. (2015). Ley de carrera docente: un punto de quiebre. PUCV.

Rodríguez M. (2010) International Journal of Sport Science, Volumen VI - Año VI, Pág: 12, No 18 - Enero - 2010, "Deporte, Salud y Calidad de Vida".

Sánchez Bañuelos, F. (1996). La actividad física orientada hacia la salud. Madrid.

Valls, A. (2003). Habilidades Directivas Claves. Ediciones Gestión 2000. Barcelona. España.

Wolff, M. (2002). Cambios epidemiológicos en las enfermedades infecciosas en Chile durante la década 1990-2000: 1990-2000. Revista médica de Chile, 130(4), 353362. 